\title{
W X Y
}

Warfarin Coumarin anticoagulant that interferes with synthesis of clotting factors by the liver. May produce allergic reactions. Overdosage produces haemorrhage controlled by Vitamin K. Potentiated by drugs such as Acetylsalicylic acid and Phenylbutazone which displace from protein binding, and reduced by hepatic enzyme inducers, such as barbiturates. Caution if used in liver disease.

Wheat husk Concentrated extract of non-absorbable fibre content of wheat. Used as bulking agent in treatment of constipation. May cause flatulence and abdominal distension.

Wool fat Fat/grease recovered from wool. Resembles the secretion from human sebaceous glands in the skin. Mixed with vegetable or soft paraffin oils it produces emollient creams which penetrate the skin and aid drug absorption through the skin. May cause skin sensitization.

Xipamide Diuretic with potency similar to Frusemide but slower onset and longer duration of action. Uses and adverse effects similar to Bendrofluazide.

Xylometazoline Sympathomimetic, used topically as nasal decongestant. Actions and adverse effects similar to Naphazoline.

Yohimbine Plant extract with alpha-adrenoceptor blocking actions. Said to have aphrodisiac properties but not proven. 